\title{
BMJ Open Effect of obesity on cognition in adults with and without a mood disorder: study design and methods
}

\author{
Maria R Restivo, ${ }^{1}$ Margaret C McKinnon, ${ }^{1,2}$ Benicio N Frey, ${ }^{1}$ Geoffrey B Hall, ${ }^{2}$ \\ Valerie $\mathrm{H}$ Taylor $^{1,3,4}$
}

To cite: Restivo MR,

McKinnon MC, Frey BN, et al. Effect of obesity on cognition in adults with and without a mood disorder: study design and methods. BMJ Open 2016;6:e009347. doi:10.1136/bmjopen-2015009347

- Prepublication history for this paper is available online To view these files please visit the journal online (http://dx.doi.org/10.1136/ bmjopen-2015-009347)

Received 8 July 2015 Revised 16 December 2015 Accepted 26 January 2016

\section{(1) CrossMark}

${ }^{1}$ Department of Psychiatry and Behavioural

Neurosciences, McMaster University, Hamilton, Ontario, Canada

2Department of Psychology, Neuroscience \& Behaviour, McMaster University, Hamilton, Ontario, Canada 'Women's College Research Institute, Women's College Hospital, Toronto, Ontario, Canada

${ }^{4}$ Department of Psychiatry, University of Toronto, Toronto, Ontario, Canada

Correspondence to Dr Valerie H Taylor; valerie.taylor@wchospital.ca

\section{ABSTRACT}

Introduction: Obesity is a common medical illness that is increasingly recognised as conferring risk of decline in cognitive performance, independent of other comorbid medical conditions. Individuals with mood disorders (bipolar disorder (BD) or major depressive disorder (MDD)) display an increased prevalence of both obesity and risk factors for cardiovascular diseases. Moreover, BD and MDD are associated with impairment in cognitive functioning across multiple domains. The independent contribution of obesity to cognitive decline in this population has not been explored. This study examines the impact of obesity on cognition by comparing neuropsychological performance in obese individuals, with or without a mood disorder before and after undergoing bariatric surgery.

Methods and analysis: This study compares measures of declarative memory, executive functioning and attention in obese individuals (body mass index $>35 \mathrm{~kg} / \mathrm{m}^{2}$ ) with BD or MDD, and 2 control populations (obese individuals without a psychiatric illness and healthy non-obese controls) prior to and following bariatric surgery. Participants (ages 18-60) receive a psychiatric diagnosis via the Structured Clinical Interview for the Diagnostic and Statistical Manual of Mental Disorders, Fourth Edition (DSM-IV; SCID). Mood ratings, physical measurements, nutritional and health questionnaires are also administered. A standardised battery of neuropsychological tests aimed at establishing performance in areas of declarative memory, executive functioning and attention are administered.

Warrington's Recognition Memory Task (RMT) and an $\mathrm{N}$-Back Task are performed in a $3 \mathrm{~T}$ functional MRI to investigate patterns of neural activation during cognitive performance. Additionally, anatomical MRI data are obtained to investigate potential changes in neural structures. Baseline data will be analysed for between-group differences and later compared with postsurgical data to investigate cognitive change.

Ethics and dissemination: This study has been approved by the Hamilton Integrated Research Ethics Board (09-3254). Results will be available in peerreviewed scientific publications and scientific meetings presentations, and released in lay form to media.

\section{Strengths and limitations of this study}

- Only known study to follow use of functional MRI (fMRI) to study memory/higher order cognitive changes before and after a significant weight status change.

- Extensive characterisation and well-controlled design of population comorbidities.

- Quantitative complimentary and comprehensive cognitive data collection methodologies (standardised neuropsychological measures, fMRI neural activation investigation and MRI neural structure measurements).

- Study entry limited by physical MRI restrictions (may capture limited portion of bariatric surgery population).

\section{BACKGROUND}

\section{Obesity and cognition}

Cognitive functions are frequently divided into the domains of perception, attention, memory and executive function, with executive function including a diverse range of higher order processes such as planning, regulation and goal-oriented behaviour. ${ }^{1}$ Each of these general categories can then be divided further into specific subtypes of $\operatorname{cog}$ nitive function; memory, for example, is commonly divided into implicit or procedural memory (skill-based memory), semantic memory (fact-based memory) and episodic memory (memory related to biographical events). These distinctions are not merely theoretical in nature, but also represent distinct neuroanatomical circuits coordinating different aspects of memory and cognition more broadly. ${ }^{2}$

The pathways through which obesity negatively affects cognition are not well elucidated. Although a number of medical conditions have been shown individually to adversely affect cognition, recent research suggests that adiposity itself may have a negative association with cognitive performance. ${ }^{34}$ 
Research focused solely on the relation between obesity (in absence of comorbid medical health conditions) and cognition is slowly emerging. In a previous meta-analysis completed by van den Berg et $a l^{3}{ }^{3}$ only six studies investigating the association between obesity and cognition were identified. ${ }^{3}$ One out of three crosssectional and two out of three longitudinal studies reported a significant negative association between obesity and cognitive performance, with this association differing across individual cognitive domains. In a recent review, Smith et a $\bar{l}$ found that 14 out of 15 crosssectional studies in human adult participants reported a negative association between obesity and cognition. Interestingly, executive functioning was the cognitive domain most often affected (11 out of 15 studies reported an association). There were only four prospective studies examining the impact of obesity and naturalistic weight changes on cognitive performance and later life outcome; the results from these four studies were inconsistent. While much of the prospective data showed that a higher body mass index (BMI) or waist-to-hip ratio was associated with poorer performance on tests of memory, Gunstad et at found that waist circumference and BMI were associated with faster performance on a neuropsychological measure of processing speed. To our knowledge, there have been two further studies published since this time also investigating the relation between obesity and cognition. ${ }^{4}$ Discrepancies among the reported results in studies of cognition and obesity may be due to the lack of consistency in study design, including heterogeneity in inclusionary baseline BMI, age of participants, present comorbidities, type of weight change (increase/decrease over time) and type of intervention applied (eg, level of dietary restriction, surgical intervention or changes in physical activity levels).

In addition, the majority of the literature examining the relation between cognition and obesity did not differentiate between the effects of obesity itself and its related comorbidities. For example, the large prospective Framingham Heart Study by Elias et al had 1423 community participants complete tests involving IQ verbal memory and verbal fluency. After adjusting for potential confounders of age, education, occupation, alcohol and smoking use, dyslipidaemia and diabetes, significant effects of hypertension and obesity were observed on tests of learning, memory and intellectual functioning in men only. The effects of hypertension and obesity were interdependent (both resulted in diminished cognitive performance, but alone were insignificant). By contrast, Kuo et al $\mathrm{s}^{9}$ study of 2684 normal weight, overweight and obese participants included completion of the Mini-Mental State Examination (MMSE), verbal learning, memory and reasoning tasks, and performance measures. After age, race, sex, intervention type, education, and cardiovascular (CV) comorbidities were controlled for, overweight participants had better overall cognitive performance on measures of verbal reasoning and processing speed. Clearly, there is an urgent need for well-designed and controlled weight loss intervention studies that can adequately assess changes in cognition following significant, maintained weight loss and monitored health changes in overweight and obese individuals.

\section{Obesity and mood disorders}

There is an estimated 12-month $9.5 \%$ prevalence of mood disorders (bipolar disorder (BD) and major depressive disorder (MDD)) in the general population, and the lifetime prevalence of mood disorders is more than double this at $20.8 \% .{ }^{10}{ }^{11}$ Individuals with mood disorders have a greater prevalence of risk factors for $\mathrm{CV}$ disease, including type 2 diabetes (T2D), smoking and hypertension (BD is also associated with an increased risk factor for hypertriglyceridaemia), ${ }^{12}$ finding that may in part be explained by the high rates of obesity in this population. The National Comorbidity SurveyReplication (NCS-R) reported ORs for obesity of 1.47 for lifetime BD and 1.21 for MDD. ${ }^{13}$

It has been well documented that both MDD and BD are associated with impairment in cognitive functioning across multiple frontal temporally mediated cognitive domains, including executive functioning, attention memory. ${ }^{14-17}$ Impairment on tests involving the conscious recollection of facts or events is among the most consistent deficit reported in patients with a mood disorder. Further, this declarative memory deficit may be most severe in patients with long-term illness duration or recurrent mood episodes. ${ }^{18}$ Studies also indicate executive function impairment on tasks involving the selection, timing, monitoring and interpretation of behaviour, including working memory and selective attention. ${ }^{19}{ }^{20}$ Although these cognitive deficits persist into the euthymic state in many patients, ${ }^{21}$ their implications for daily functioning are not fully understood. ${ }^{22}$ Critically, the presence of cognitive impairments, in particular, deficits in executive functioning and in verbal memory, has been associated with poor functional outcomes (eg, vocational) in patients with mood disorders. ${ }^{23-30}$

Cognitive dysfunction is not always saliently present at the time of illness onset in mood disorders, often emerging over the course of illness and worsening with illness duration. ${ }^{14}$ This may be attributed, in part, to a combination of clinical and treatment variables. Antidepressants (especially antidepressants that target more than one receptor) for example, are associated with improvements in some aspects of cognition, while other areas may be more resistant. ${ }^{31}$ Some psychotropic medications used in the treatment of BD have also been associated with $\operatorname{cog}$ nitive improvement ${ }^{19}$ and lithium, which is widely used as a mood stabiliser, has been shown to have a neuroprotective effect resulting in neurogenesis in the hippocampus (an important neural structure in memory processing).$^{32}$ In contrast, however, the chronic effects of anticholinergic drugs have been shown to increase risk for permanent cognitive impairment. ${ }^{33}$ Adding to 
this issue are clinical variables, as early onset MDD itself is associated with increased risk for Alzheimer's disease. $^{34}$

Fortunately, there is evidence that cognition may be amenable to strategies aimed at preventing or reducing functional impairment. Recent studies suggest that cognitive remediation approaches (eg, computerised skills training) may improve cognitive functioning in patients with mood disorders. ${ }^{35}{ }^{36}$ However, residual cognitive symptoms often persist in patients with euthymia. ${ }^{21}$ Also, many medications used in treating mood disorders are also associated with increased weight gain and related metabolic comorbidities; this weight gain and metabolic dysregulation can be quite severe with certain medication classes (such as atypical antipsychotics). Moreover, these metabolic changes may themselves be associated with cognitive impairment in areas of memory and executive function. ${ }^{37}$ Thus, it may be that the cognitive improvement expected in medicated or treated mood disorder patients is negated over time, and may ultimate manifest as cognitive decline, as a consequence of this associated weight gain. ${ }^{37}$ Given that a strong association between cognitive impairment and poor psychosocial functional outcomes has been established, understanding the interaction between medication use, weight and cognition is of great concern to treating practitioners. ${ }^{15}$

\section{Study objective}

The goal of this study is to examine the impact of obesity on memory, executive function and attention in patients with and without a mood disorder (MDD or $\mathrm{BD})$ by assessing cognitive performance prior to, and after, a significant 1-year weight loss following bariatric surgery. Changes in cognition associated with weight loss have been difficult to investigate primarily because most weight loss interventions do not result in a significant weight change. ${ }^{39}$ We are uniquely positioned to investigate this, however, as we have designed an assessment paradigm that focuses on bariatric surgery patients. Bariatric surgery results in a weight loss range of $12-39 \%$ of presurgical body weight, providing an effective intervention with which to assess cognitive change. ${ }^{40}$ We have also worked with engineers to modify our MRI to accommodate physical restrictions associated with cognitive testing in this population, allowing us to examine some of the brain correlates behind this association. The specific aims and hypotheses of the study are:

\section{Aim 1: Determine the effect of obesity (and additional interactive effect of a mood disorder diagnosis) on cognitive performance.}

Hypothesis 1a: Compared with a healthy BMI weight nonpsychiatric control population, the obese (bariatric) non-psychiatric control population will show worse cognitive performance, as assessed by the outcome of a standardised cognitive battery, prior to bariatric surgery.
Hypothesis 1b: Obese (bariatric) patients with a $\mathrm{BD}$ or MDD diagnosis will show worse cognitive performance than both (healthy BMI and obese (bariatric)) control populations, as assessed by the outcome of a standardised cognitive battery, prior to bariatric surgery.

Aim 2: Examine whether structural or functional brain differences can be seen (either in neural activation patterns during cognitive tasks or structurally) in obese patients with or without a mood disorder

Hypothesis 2: Prior to surgery, bariatric groups will show differences in regional pattern activations relative to the healthy BMI weight control group in neural activation during declarative memory and executive functioning tasks. These regional differences will be seen in neural structures important to memory (such as the hippocampus and precuneus) and executive function, such as the dorsolateral prefrontal cortex.

Aim 3: Investigate whether any differences seen and associated with obesity (in cognitive performance tasks, neural activation patterns or neural structures) can be diminished following significant weight loss

Hypothesis 3: At 1-year postintervention, all surgery-treated groups will show a significant improvement in cognitive performance measures (and related neural investigations) following expected (12-39\% presurgical body weight ${ }^{40}$ ) weight loss and overall health improvement.

The overall goal of this project is to quantify cognitive impairment in patients with mood disorders and assess the impact of obesity on cognitive performance and brain activation by measuring these variables before and after an intervention that significantly alters weight. We speculate that changes in cognitive function associated with mood disorders are caused in part by weight status, thereby increasing the burden of illness associated with MDD and BD.

\section{METHODS}

\section{Study design and timeline}

This is a prospective cohort study. Study participants are seen 2-4 times during the study. Prior to surgery, participants are seen once to complete cognitive testing and once to complete the brain imaging session. They will be required to return for a second cognitive testing and functional MRI (fMRI) session 1 year after surgical intervention (or 13 months following baseline visits for healthy control participants). Self-report questionnaires, psychiatric assessments and anthropomorphic measures are administered at the presurgical and postsurgical time points as well.

\section{Participants: recruitment, screening and enrolment}

Bariatric participants are recruited from the St. Joseph's Healthcare Hamilton programme for Bariatric Surgery (an Ontario Centre for Surgical Excellence). All patient charts on file were manually screened for potential 
eligibility in an initial recruitment stage; potential eligibility was based on reported patient height and weight measurements, age and whether the patient was still awaiting surgery. Newly received referral patients continue to be screened on an ongoing basis. Patients deemed potentially eligible are first reached via telephone. The study is introduced and procedures are explained during this initial telephone contact; if interested, participants then undergo a telephone screen to determine if they meet study inclusion/exclusion criteria. Those who meet criteria are then scheduled for baseline study appointments and written informed consent is obtained at the initial appointment prior to data collection. Healthy control participants from a departmental consent-to-contact phone list are contacted via telephone and administered parallel screening and enrolment procedures. In addition, recruitment also occurs via advertisements placed on hospital notice boards, and from healthcare provider referrals. Bariatric participants can be enrolled in the study during any stage following the orientation class of their presurgical process. The surgical candidacy process (and estimate time intervals between candidacy stages) can be found in figure 1 .

Study participants are recruited into four groups: obese (bariatric) patients with $\mathrm{BD}$, obese (bariatric) patients with MDD, obese (bariatric) patient without a psychiatric disorder (past or present) and healthy weight (non-surgical) controls without a psychiatric disorder (past or present). Inclusion criteria for all groups are as follows: age 18-60 years, ability to provide informed consent and native English speaker (or having learned English by age 6). Additionally, healthy controls are required to have a BMI between 18.5 and 24.9 (normal range). Exclusion criteria include the presence of a current or pre-existing neurological condition (eg, epilepsy, severe head trauma) or unstable and/or severe medical condition (eg, cancer, life-threatening myocardial infarction), contraindications to MRI (deemed unsafe to complete an MRI via safety screening questionnaire), left handedness (confirmed via Edinburgh
Handedness Inventory), ${ }^{41}$ having been administered any of the cognitive study measures within the past 12 months, a history of a confirmed learning disorder or developmental disability diagnosis (eg, attention deficit hyperactivity disorder) or a Full Scale IQ (FSIQ) $<70$, an inability to complete the testing (eg, due to a hearing or vision impediment), and the presence of alcohol or substance abuse within the past 6 months or lifetime dependency (those in the BD group will not be excluded due to lifetime dependency if in sustained full remission). In addition, presence of a past or current psychiatric condition is an exclusion criterion for both healthy BMI weight and bariatric (obese) nonpsychiatric control groups while having been administered electroconvulsive therapy within the past 24 months is an exclusion criterion for both BD and MDD bariatric patient groups. MRI eligibility screening is independently performed by MRI technicians at the Imaging Research Centre (St. Joseph's Healthcare Hamilton, Ontario, Canada). Participants who are unable to complete MRI testing but have completed all other testing remain enrolled in the study. The first study participant was enrolled on 22 September 2010.

\section{Surgical intervention}

Currently in Ontario, there are 150000 individuals eligible for bariatric surgery and over 3000 individuals actively pursuing bariatric surgery. As of 2014, the Bariatric Surgery Program at St. Joseph's Healthcare Hamilton completed approximately 600 surgeries per year. ${ }^{42}$ Traditionally, all bariatric surgeries have been thought to cause weight loss through the processes of malabsorption (of nutrients or calories), caloric restriction or a combination of the two. ${ }^{40}$

The most common gastric procedures performed are laparoscopic adjustable gastric banding (LAGB) and Roux-en-Y gastric bypass (RYGB) ${ }^{43}$ In Ontario, RYGB is the most routinely performed and is covered financially (for those with a BMI exceeding 40 or 35 with significant medical comorbidities) by the Ontario Health

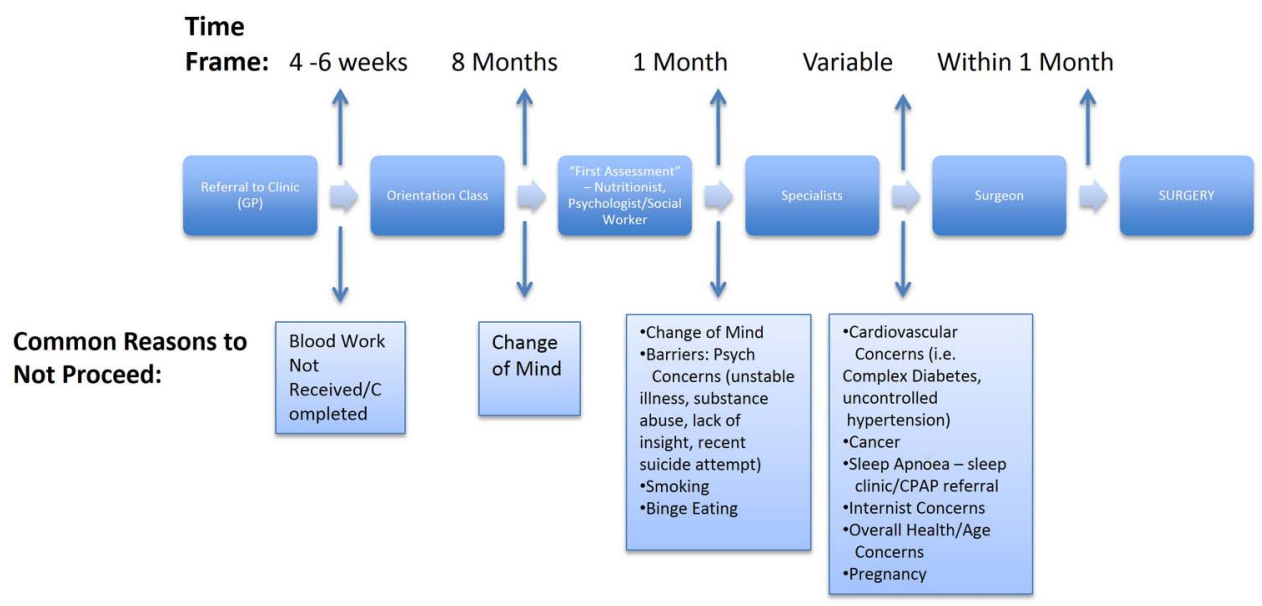

Figure 1 The surgical candidacy process and estimate time intervals between candidacy stages. CPAP, continuous positive airway pressure; GP, general practitioner. 
Insurance Program. Alternatively, the LAGB is rarely performed in public health settings due to its diminished rate of long-term weight loss success and the higher likelihood for additional follow-up surgical procedures; it is, however, readily available through private healthcare providers. Owing to the presence of certain medical comorbidities, conditions or gastrointestinal irregularities, a bariatric surgery team may opt to perform a laparoscopic vertical sleeve gastrectomy or biliopancreatic diversion with duodenal switch. ${ }^{44}$

\section{Data collection}

Participants complete baseline measures over the course of 1-2 study visits. Those who undergo MRI at baseline are reassessed for scan eligibility at their follow-up visit. All assessment measures are readministered at follow-up with the exception of the Structured Clinical Interview for Diagnostic and Statistical Manual of Mental Disorders, Fourth Edition (DSM-IV; SCID) (which is replaced by the Mini International Neuropsychiatric Interview (MINI) at that time point). A double-entry system with independent research personnel is utilised for all cognitive and behavioural data, and inconsistencies are checked and resolved by an additional assessor. A summary of the test measures administered at each time point is found in table 1 . To accommodate patient schedule restrictions, study visits 1 and 2 (presurgical baseline), and study visits 3 and 4 (postsurgical follow-up) may occasionally be collapsed into one visit. Similarly, self-report questionnaires and psychiatric measures may occasionally be administered on the same study visit to accommodate the patient.

\section{Psychiatric (and mood) assessment}

Participants are diagnosed via administration of the SCID at baseline. Current psychiatric status is reassessed at follow-up via the MINI. Both current and euthymic patients are included in the bariatric $\mathrm{BD}$ and bariatric MDD groups. As this is not an intervention trial, patients are not assigned nor treated by a study psychiatrist. Patients currently being treated by a community or clinic mental health professional or general practitioner will continue to do so. If patients indicate symptoms of current suicidality, they are referred to emergency psychiatric services. Mood ratings are also monitored at baseline and end visits via the Hamilton Rating Scale for Depression (HAMD-17) and the Young Mania Rating Scale (YMRS) $;{ }^{45}$ both are assessed by the same study personnel to avoid issues of inter-rater reliability. In addition, the Beck Depression Inventory (BDI) and Altman Self-Rating Scale for Mania (ASRM) are also administered. ${ }^{47}$ In circumstances where baseline visits are 2 or more weeks apart, the BDI and ASRM are administered separately at each of these visits to account for possible changes in mood state. As high rates of trauma exposure have been reported in both mood disorder and obese populations, ${ }^{49} 50$ the Childhood Trauma Questionnaire (CTQ) is also administered. ${ }^{51}$

\section{Neuropsychological assessment}

A standardised battery of neuropsychological tests aimed at establishing preintervention and postintervention performance on tests of declarative memory, executive functioning and attention is administered. These cognitive domains have been shown to be susceptible to impairment in metabolically dysregulated populations. ${ }^{3} 8$ Executive function is also shown to be the most robust cognitive domain susceptible to impairment in obese populations specifically, ${ }^{52}$ while there is also evidence indicating the areas of memory ${ }^{53-55}$ and attention ${ }^{6} 5657$ may also be affected. Tests were chosen with two objectives in mind: (1) to investigate different aspects of both declarative memory and executive functioning in order to provide an exhaustive overview of these composite areas, and (2) with redundant overlap between areas and skills tested (to minimise the likelihood of spurious test results in any one subdomain). Additional information regarding individual neuropsychological tests administered is also summarised in table 2. A clinical neuroscience graduate student (MRR), trained in neuropsychological assessment and psychometric methodologies, administers the testing. She has received training and is supervised by a registered clinical neuropsychologist (MCM).

Declarative memory function battery:

1. California Verbal Learning Test II (standard and alternate forms): this word list learning task provides indices of immediate and delayed memory performance, interference learning, and recognition. ${ }^{64}$

2. Wechsler Memory Scale III-Logical Memory subtest: this contextually based memory task provides indices of learning slope, immediate and delayed memory performance, retention, and recognition. ${ }^{65}$

3. Brief Visuospatial Memory Test-Revised: a nonverbal test of visuospatial memory under explicit encoding conditions. ${ }^{66}$

Executive functioning and attention battery:

1. Controlled Oral Word Association Task (COWAT): this task taps phonemic and semantic (animals) fluency. ${ }^{67}$

2. Stroop Colour and Word Test (Golden version): this task taps sensitivity to suppress a habitual response in favour of a less familiar one. ${ }^{68}$

3. Wisconsin Card Sorting Task (64-item version): this task taps the ability to form and shift concepts based on feedback. ${ }^{69}$

4. Colour Trails Test Part A and B: whereas Part A assesses processing speed, Part B taps the ability to sequence two stimulus sets while alternating between them. ${ }^{70}$

5. Paced Auditory Serial Attention Test (Victoria Computerized Adaptation): this task assesses capacity and rate of information processing as well as sustained and divided attention. ${ }^{71}$

\section{Premorbid IQ}

Participants complete one subtest of the performance (matrix reasoning) and verbal (vocabulary) indices of 
Table 1 Study visit schedule

\begin{tabular}{|c|c|c|c|c|}
\hline & $\begin{array}{l}\text { First visit (baseline/ } \\
\text { screening, presurgical) }\end{array}$ & $\begin{array}{l}\text { Second visit } \\
\text { (presurgical) }\end{array}$ & $\begin{array}{l}\text { Third visit } \\
\text { (1 year following } \\
\text { surgery) }\end{array}$ & $\begin{array}{l}\text { Fourth visit } \\
\text { (follow-up, } \\
\text { postsurgical) }\end{array}$ \\
\hline SCID (DSM-IV-TR) & $\mathbf{x}$ & & & \\
\hline MINI & & & $\mathbf{x}$ & \\
\hline HAMD-17 & $\mathbf{x}$ & & $\mathbf{x}$ & \\
\hline YMRS & $\mathbf{x}$ & & $\mathbf{x}$ & \\
\hline BDI & $\mathbf{x}$ & & $\mathbf{x}$ & \\
\hline ASRM & $\mathbf{x}$ & & $\mathbf{x}$ & \\
\hline Anthropomorphic, glucose measures & $\mathbf{x}$ & & $\mathbf{x}$ & \\
\hline Wechsler Abbreviated Scale of Intelligence & $\mathbf{x}$ & & $\mathbf{x}$ & \\
\hline The Wechsler Test of Adult Reading & $\mathbf{x}$ & & $\mathbf{x}$ & \\
\hline fMRI & & $\mathbf{x}$ & & $\mathbf{x}$ \\
\hline Practice session & & $\mathbf{x}$ & & $\mathbf{x}$ \\
\hline Warrington's Recognition Memory Task & & $\mathbf{X}$ & & $\mathbf{x}$ \\
\hline Executive functioning (N-Back Task) & & $\mathbf{x}$ & & $\mathbf{x}$ \\
\hline Declarative memory function battery & & & & \\
\hline $\begin{array}{l}\text { California Verbal Learning Test II (standard } \\
\text { and alternate forms) }\end{array}$ & $\mathbf{x}$ & & $\mathbf{x}$ & \\
\hline Wechsler Memory Scale III-Logical & $\mathbf{x}$ & & $\mathbf{x}$ & \\
\hline \multicolumn{5}{|l|}{ Memory subtest } \\
\hline $\begin{array}{l}\text { Brief Visuospatial Memory Test-Revised } \\
\text { Executive functioning and attention battery }\end{array}$ & $\mathbf{x}$ & & $\mathbf{x}$ & \\
\hline Controlled Oral Word Association Task & $\mathbf{x}$ & & $\mathbf{x}$ & \\
\hline $\begin{array}{l}\text { Stroop Colour and Word Test (Golden } \\
\text { version) }\end{array}$ & $\mathrm{x}$ & & $\mathbf{x}$ & \\
\hline $\begin{array}{l}\text { Wisconsin Card Sorting Task (64-item } \\
\text { version) }\end{array}$ & $\mathbf{x}$ & & $\mathbf{x}$ & \\
\hline Colour Trails Test Part A and B & $\mathbf{x}$ & & $\mathbf{x}$ & \\
\hline Paced Auditory Serial Attention Test & $\mathbf{x}$ & & $\mathbf{x}$ & \\
\hline \multicolumn{5}{|l|}{ Additional self-report questionnaires } \\
\hline Cognitive Failure Questionnaire & & $\mathbf{X}$ & & $\mathbf{x}$ \\
\hline Sheehan Disability Questionnaire & & $\mathbf{x}$ & & $\mathbf{x}$ \\
\hline $\begin{array}{l}\text { Berlin Sleep Questionnaire (healthy } \\
\text { controls only) }\end{array}$ & & $\mathbf{x}$ & & $\mathbf{x}$ \\
\hline Childhood Trauma Questionnaire & & $\mathbf{x}$ & & $\mathbf{x}$ \\
\hline Demographics Questionnaire & & $\mathbf{x}$ & & $\mathbf{x}$ \\
\hline Food Frequency Questionnaire* & $\mathbf{x}$ & & $\mathbf{x}$ & \\
\hline Medications List Questionnaire* & $\mathbf{x}$ & & $\mathbf{x}$ & \\
\hline $\begin{array}{l}\text { *Both these questionnaires require extensive informa } \\
\text { food intake). As such, they are given to study particip } \\
\text { study visit. } \\
\text { ASRM, Altman Self-Rating Scale for Mania; BDI, Bec } \\
\text { Disorders, Fourth Edition, Text Revision; fMRI, functi } \\
\text { Neuropsychiatric Interview; SCID, Structured Clinical }\end{array}$ & $\begin{array}{l}\text { onal MRI; HAMD, Hamilton Rat } \\
\text { Interview for DSM-IV; YMRS, }\end{array}$ & $\begin{array}{l}\text { Questionnaire re } \\
\text { th time points to }\end{array}$ & $\begin{array}{l}\text { iring a minimum 3-day } \\
\text { e completed and returr }\end{array}$ & $\begin{array}{l}\text { recording of } \\
\text { ed by their next } \\
\text { of Mental } \\
\text { national }\end{array}$ \\
\hline
\end{tabular}

the Wechsler Abbreviated Scale of Intelligence in order to estimate current intellectual functioning via FSIQ. ${ }^{72}$ The Wechsler Test of Adult Reading is also administered to estimate premorbid intellectual functioning in participants. ${ }^{73}$ This test consists of 50 words, listed in order of difficulty. Participants are presented with the word list and instructed to read each word aloud. Total number of correct pronunciations comprises the final score.

\section{Anthropometric measures}

A glucose measurement is obtained on the day of cognitive testing both at baseline and end visits via a 'finger prick' glucometer reading. Height, weight, waist and hip circumferences, systolic and diastolic blood pressure, and heart rate measurements are measured for nonbariatric controls at baseline and end visits. Waist circumference is measured according to the WHO STEPS protocol that instructs that the measurement is made approximately between the lower margin of the last palpable rib and the top of the iliac crest. ${ }^{74}$ Hip circumference is measured around the widest portion of the participant's buttocks.

For bariatric surgery participants, these measurements are obtained via manual data extraction by study personnel from participants' medical record charts containing doctor, nurse and dietician visit notes and summary. 
Table 2 Summary and psychometric properties of neuropsychological test measures and functional MRI (fMRI) behavioural tasks $^{58}$

\begin{tabular}{|c|c|c|c|}
\hline Test & $\begin{array}{l}\text { Administration time } \\
\text { (minutes) }\end{array}$ & $\begin{array}{l}\text { Age range } \\
\text { (years) }\end{array}$ & Measure and purpose \\
\hline $\begin{array}{l}\text { Brief Visuospatial Memory } \\
\text { Test-Revised (BVMT-R) }\end{array}$ & $\begin{array}{l}15 \text { ( } 40 \text { with delay } \\
\text { interval) }\end{array}$ & $18-79$ & $\begin{array}{l}\text { Multiple trial figure-learning paradigm assessing } \\
\text { visual learning and memory }\end{array}$ \\
\hline $\begin{array}{l}\text { California Verbal Learning } \\
\text { Test-II (CVLT-II) }\end{array}$ & $35-40$ & $16-89$ & $\begin{array}{l}\text { Multiple trial list-learning paradigm assessing verbal } \\
\text { learning and memory }\end{array}$ \\
\hline Color-Trails Test (CTT) & $5-10$ & $18-89$ & $\begin{array}{l}\text { Manual drawing task assessing speed of attention, } \\
\text { sequencing, mental flexibility, visual search and } \\
\text { motor function }\end{array}$ \\
\hline N-Back Task & 22 (fMRI version) & Not defined & $\begin{array}{l}\text { Continuous performance task assessing attention } \\
\text { and short-term memory }\end{array}$ \\
\hline $\begin{array}{l}\text { Paced Auditory Serial Addition } \\
\text { Task (computerised version) }\end{array}$ & $15-20$ & $16-74$ & $\begin{array}{l}\text { Serial addition task assessing working memory, } \\
\text { divided attention and information processing speed }\end{array}$ \\
\hline Stroop (Golden version) & 5 & $5-90$ & $\begin{array}{l}\text { Reading task assessing cognitive control, goal } \\
\text { maintenance and suppression of a habitual } \\
\text { response in favour of a less familiar one }\end{array}$ \\
\hline $\begin{array}{l}\text { Warrington's Recognition Memory } \\
\text { Task (words subtest only) }\end{array}$ & 8 (fMRI version) & $18-70$ & Assesses recognition memory for printed words \\
\hline $\begin{array}{l}\text { Wechsler Memory Scale-III } \\
\text { (WMS-III; Logical Memory I and II } \\
\text { subtests) }\end{array}$ & $\begin{array}{l}35-45 \text { min with delay } \\
\text { interval }\end{array}$ & 18-89 & $\begin{array}{l}\text { Assesses auditory declarative (verbal) memory and } \\
\text { learning }\end{array}$ \\
\hline $\begin{array}{l}\text { Wechsler Abbreviated Scale of } \\
\text { Intelligence (WASI) }\end{array}$ & 15 & $6-89$ & Brief intelligence measure \\
\hline $\begin{array}{l}\text { Wechsler Test of Adult Reading } \\
\text { (WTAR) }\end{array}$ & 10 & $16-89$ & Reading task assessing premorbid functioning \\
\hline $\begin{array}{l}\text { Wisconsin Sorting Card Task } \\
\text { (WSCT) }\end{array}$ & $15-30$ & $5-89$ & $\begin{array}{l}\text { Card-sorting task assessing ability to form abstract } \\
\text { concepts, shift and maintain set, and utilise } \\
\text { feedback }\end{array}$ \\
\hline
\end{tabular}

Nurses and dieticians review and record relevant blood work that must be completed and accessible by the patient's first clinical visit, and record the anthropometric measures described earlier (such as blood pressure, weight and waist circumference) during this first visit. Glucose, glycated haemoglobin and lipid assessment profiles contained in the participants' medical record for bariatric participants were obtained via data extraction by study personnel from laboratory reports.

\section{Demographics and medical health}

Age, gender, education, job status, family psychiatric history and medical health/illness information is collected during the initial telephone screen questionnaire. As part of the bariatric surgery process, clinic staff capture extensive information regarding the patient's past and current medical diagnoses during the patient's initial clinic visit and study personnel extract data recorded during these encounters in order to confirm the presence or absence of comorbidities. It is expected that the vast majority of enrolled participants will receive Roux X-en-Y gastric bypass; however, given that different surgical procedures are associated with different rates and mechanisms of weight loss and that a minority of patients may receive an alternate surgery, the type of bariatric surgery completed by each participant is also recorded. This will allow us to investigate the potential variation in outcomes for patients receiving different forms of surgery. Additional information concerning living arrangements, previous education details, marital/ relationship status, number of children, smoking behaviour and previous medication history is collected in the general demographics questionnaire administered during the study.

Participants are also asked to provide a complete listing of current medications, vitamins and herbal supplements (including dosage and indication), at both baseline and follow-up time points. The Berlin Sleep Questionnaire, ${ }^{75}$ which assesses the risk level for current obstructive sleep apnoea (OSA) or sleep-disordered breathing is also completed. It is administered as part of the study self-report package for non-bariatric participants, while bariatric participants complete this questionnaire through the bariatric surgery clinic as part of their surgical candidacy process. OSA will be analysed as a categorical variable (present, current controlled through treatment or absent). OSA status is reassessed at follow-up as well. Patients complete a self-report questionnaires regarding their current level of physical activity and mobility (both presurgically and postsurgically). 
Increasing levels of physical activity has been associated with positive cognitive outcomes and increased hippocampal size. ${ }^{34} 76$ Although not a standardised, quantitative questionnaire, this self-report may provide us with a qualitative assessment of current level of exercise that can be converted into a categorical variable representing general level of physical activity (eg, sedentary, minimal, moderate or high) to be used in later exploratory analyses.

\section{Nutrition}

Nutritional intake is assessed via a non-consecutive 3-day dietary record (Food Frequency Questionnaire), with 1 day being a weekend day. ${ }^{78}$ This 3 -day method has been demonstrated to estimate habitual energy intake within $10 \%$ of the actual values in groups as small as 13 participants. ${ }^{79}$ In addition to overall caloric intake, diet component analysis will also be completed. Specifically, total and per cent intake of proteins, carbohydrates, fat, cholesterol, fibre, sugar and sodium is calculated per participant for future analysis.

\section{Disability and self-reported cognitive measures}

The Sheehan Disability Scale (SDS) ${ }^{80}$ is administered to provide a quick measure of the impact of the participant's disability (obesity and/or mood disorder) across various life domains. The Cognitive Failure Questionnaire, ${ }^{81}$ a measure of self-reported failures in perception, memory and motor function that has been used in comparable populations previously, ${ }^{82}$ is used to assay subjective feelings of cognitive dysfunction.

\section{Imaging}

Each participant also undergoes a $1 \mathrm{~h}$ MRI session at baseline and follow-up time points. A high-resolution axial three-dimensional (3D) anatomical T1-weighted scan with full brain coverage is performed to obtain relevant neuroanatomical data (including hippocampus volume). Anatomical data collected will also allow for exploratory whole brain analysis (eg, global volume differences) to be completed. Following this, two tasks tapping declarative memory function (Warrington's Recognition Memory Task, or RMT) ${ }^{83}$ and executive functioning (N-Back Task) are performed (additional information regarding Warrington's RMT the N-Back Task is available in table 2). Regional activation patterns will be compared and contrasted across groups. Behavioural data, such as reaction time, correct number of responses on N-Back subtests and correct number of recognition hits on the RMT, are also collected. As part of the participant's orientation and training, practice trials of each task are administered outside of the MRI on the day of the actual MRI session.

\section{Data analysis}

R Statistical Software (R: A Language and Environment for Statistical Computing (program). Vienna, Austria: R Foundation for Statistical Computing, 2014) and the
Statistical Package for Social Sciences (SPSS) statistics will be used (IBM SPSS Statistics. V.22 (program), 2013) for data analysis. MRI analyses will be completed using Statistical Parametric Mapping (SPM), Matlab (MATLAB and Statistics Toolbox Release 2012b (program). Natick, Massachusetts, USA2012) and FreeSurfer (FreeSurfer V.1.0 (program). Boston, Massachusetts, USA, 2011).

Cognitive performance on neuropsychological measures at both baseline and end visits will be compared across four groups (bariatric MDD, bariatric BD, bariatric controls and healthy matched controls). The primary outcome variable at follow-up will be cognitive change at 12 months following surgery. We chose group sample sizes of 20 minimum in order to have enough power to adequately examine neuroimaging differences between groups. ${ }^{84}$ Based on work by Woods, ${ }^{85}$ we will also have enough power for use of individual contrast images in second-level random-effects models that will allow us to investigate target regional responses at the group level.

Neuropsychological measures will be examined independently and may be integrated into an executive function/attention composite and declarative memory composite. Composite score may be obtained by converting individual scale scores across to z-scores and then averaging across independent measures.

Exploratory analyses using descriptive statistics will be used to present demographic and medical data (such as comorbidity presence, age, physical activity, medication load, etc). Initial one-way between-group univariate analyses of variance will be run to identify potential confounding covariates in any effects found at baseline. The impact of related comorbidities (such as T2D and hypertension) will also be examined. Although our primary interest is the effect of obesity alone on cognition, additional CV comorbidities are likely to have an additive effect on cognitive performance and their effect contribution will be explored via hierarchal regression model analysis. Bariatric surgery is known to normalise blood glucose and reverse T2D status in surgery patients even without significant weight loss. ${ }^{40}$ This potential effect on overall cognitive performance differences will be explored in postsurgical group analyses.

Both structural and functional imaging scans will be run at baseline and follow-up using the same $3 \mathrm{~T}$ General Electric (General Electric, Milwaukee, Wisconsin, USA) system at the Imaging Research Centre (St. Joseph's Healthcare Hamilton, Ontario, Cannada). fMRI tasks will be displayed using E-Prime software (http://www.pstnet. com) (E-Prime 2.0 Software (program). Pittsburgh, Pennsylvania, USA, 2012). Hippocampal volume (and change in volume over time) will be measured using FreeSurfer (FreeSurfer V.1.0 (program). Boston, Massachusetts, USA, 2011). Acquired functional images will be processed and analysed using Statistical Parametric Mapping (SPM) and Matlab software (MATLAB and Statistics Toolbox Release 2012b (program). Natick, Massachusetts, USA, 2012). Collected 
data will be slice-time corrected, 3D motion corrected and realigned to the fifth volume in the first series collected, and normalised to Montreal Neurological Institute space. High-resolution T1-weighted 3D anatomical MRI data collected for each participant will be used for co-registration with functional data. Anatomical data sets will be averaged across healthy control participants to generate a composite image onto which the functional activation results are projected. General linear models will be created for both tasks and overlaid for each participant to examine neural activation patterns for each group. Activation contrasts will be examined using participant group as a between-participants factor.

\section{Ethics and dissemination}

Written informed consent is obtained from each participant after study information is provided and before study entry. Participants are informed that all data collected are de-identified and that identifying consent forms are kept separately from other collected data. Collected data are stored securely in both electronic and paper forms. Only approved research personnel and study investigators have access to the data. Results will be available in peer-reviewed scientific publications and scientific meetings presentations, and released in lay form to media outlets.

\section{DISCUSSION}

This study will be the first of its kind to investigate the impact of obesity on cognition via an intervention that results in significant and sustained weight loss in a population with a mood disorder. We hypothesise that weight status will have a significant effect on cognition, a conclusion that may influence the way mental healthcare is provided and have important ramifications for first-line recommendations with respect to medications. It will also improve our understanding of the neural pathways involved in cognitive processes, furthering our understanding of how mental illness develops and the additional risk conferred by obesity.

\section{Study status}

The status of the study at the time of manuscript submission was completion of enrolment for all but one participant group (bariatric BD). Numbers of participants that have been enrolled and fully completed testing in each arm of the study are available in table 3 below.

\begin{tabular}{lll} 
Table 3 & Current participant enrolment \\
\hline Group & Neuropsychology arm & MRI arm \\
\hline Healthy controls & 20 & 20 \\
Bariatric controls & 25 & 20 \\
Bariatric MDDs & 21 & 23 \\
Bariatric BDs & 11 & 4 \\
\hline BD, bipolar disorder; MDD, major depressive disorder.
\end{tabular}

Contributors MCM, MRR and VHT designed the study protocol. MRR completed study participant screening and recruitment, all data collection (including data extraction from patient records), supervised data entry (completed by undergraduate students) and completed data cleaning and coding. Thorough training under a team of clinical neuropsychologists was provided to MRR prior to start of patient testing. MCM provided feedback and consultation on cognitive data collection and analysis. GBH and BNF designed aspects of the study related to the MRI and provided feedback and consultation on MRI data collection and analysis. MRR will analyse data under the supervision of VHT and in consultation with a statistician at the Sunnybrook Health Sciences Centre (Dr Alex Kiss). MRR drafted the manuscript. All authors contributed to and approved the final manuscript.

Funding VHT received an unrestricted educational grant from Bristol Myers Squibb to help fund this study. MRR is a doctoral thesis candidate who has been supported by fellowships from the Canadian Institute of Health Research and Government of Ontario.

Competing interests None declared.

Patient consent Obtained.

Ethics approval Hamilton Integrated Research Ethics Board of St. Joseph's Healthcare Hamilton Hospital and Hamilton Health Sciences Centre (09-3254).

Provenance and peer review Not commissioned; externally peer reviewed.

Open Access This is an Open Access article distributed in accordance with the Creative Commons Attribution Non Commercial (CC BY-NC 4.0) license, which permits others to distribute, remix, adapt, build upon this work noncommercially, and license their derivative works on different terms, provided the original work is properly cited and the use is non-commercial. See: http:// creativecommons.org/licenses/by-nc/4.0/

\section{REFERENCES}

1. Sternberg RJ. Mechanisms of cognitive development: a computational approach. In: Sternberg RJ, ed. Mechanisms of cognitive development. New York, NY: Freeman, 1984: 164-86.

2. Schacter DL. Memory. In: Posner MI, ed. Foundations of cognitive science. Cambridge, MA: MIT Press, 1989:683-724.

3. van den Berg E, Kloppenborg RP, Kessels RP, et al. Type 2 diabetes mellitus, hypertension, dyslipidemia and obesity: a systematic comparison of their impact on cognition. Biochim Biophys Acta 2009;1792:470-81.

4. Fagundo $A B$, de la Torre R, Jiménez-Murcia $S$, et al. Executive functions profile in extreme eating/weight conditions: from anorexia nervosa to obesity. PLOS ONE 2012;7:e43382.

5. Smith E, Hay P, Campbell L, et al. A review of the association between obesity and cognitive function across the lifespan: implications for novel approaches to prevention and treatment. Obes Rev 2011:12:740-55.

6. Gunstad J, Lhotsky A, Wendell CR, et al. Longitudinal examination of obesity and cognitive function: results from the Baltimore Longitudinal Study of Aging. Neuroepidemiology 2010;34: 222-9.

7. Ariza M, Garolera M, Jurado MA, et al. Dopamine genes (DRD2/ ANKK1-TaqA1 and DRD4-7R) and executive function: their interaction with obesity. PLOS ONE 2012;7:e41482.

8. Elias MF, Elias PK, Sullivan LM, et al. Lower cognitive function in the presence of obesity and hypertension: the Framingham heart study. Int J Obes Relat Metab Disord 2003;27:260-8.

9. Kuo HK, Jones RN, Milberg WP, et al. Cognitive function in normal-weight, overweight, and obese older adults: an analysis of the Advanced Cognitive Training for Independent and Vital Elderly cohort. J Am Geriatr Soc 2006;54:97-103.

10. Kessler RC, Akiskal HS, Ames M, et al. Prevalence and effects of mood disorders on work performance in a nationally representative sample of U.S. workers. Am J Psychiatry 2006;163:1561-8.

11. Kessler RC, Berglund $P$, Demler $O$, et al. Lifetime prevalence and age-of-onset distributions of DSM-IV disorders in The National Comorbidity Survey Replication. Arch Gen Psychiatry 2005;62:593-602.

12. Fiedorowicz JG, He J, Merikangas KR. The association between mood and anxiety disorders with vascular diseases and risk factors in a nationally representative sample. J Psychosom Res 2011;70:145-54. 
13. Simon GE, Von Korff MS, Saunders K, et al. Association between obesity and psychiatric disorders in the US adult population. Arch Gen Psychiatry 2006;63:824-30.

14. Daglas $\mathrm{R}$, Yucel $\mathrm{M}$, Cotton $\mathrm{S}$, et al. Cognitive impairment in first-episode mania: a systematic review of the evidence in the acute and remission phases of the illness. Int J Bipolar Disord 2015;3:9.

15. Bora $\mathrm{E}$, Harrison BJ, Yücel $\mathrm{M}$, et al. Cognitive impairment in euthymic major depressive disorder: a meta-analysis. Psychol Med 2013:43:2017-26.

16. Papakostas Gl. Cognitive symptoms in patients with major depressive disorder and their implications for clinical practice. J Clin Psychiatry 2014;75:8-14.

17. Torres I, Sole B, Vieta E, et al. Neurocognitive impairment in the bipolar spectrum. Neuropsychiatry 2012;2:43.

18. MacQueen GM, Galway TM, Hay J, et al. Recollection memory deficits in patients with major depressive disorder predicted by past depressions but not current mood state or treatment status. Psychol Med 2002;32:251-8.

19. Landro NIPD, Stiles TCPD, Sletvold HPD. Neuropsychological function in nonpsychotic unipolar major depression. Neuropsychiatry Neuropsychol Behav Neurol 2001;14:233-40.

20. Harvey PO, Le Bastard G, Pochon JB, et al. Executive functions and updating of the contents of working memory in unipolar depression. $J$ Psychiatr Res 2004;38:567-76.

21. Paradiso S, Lamberty GJ, Garvey MJ, et al. Cognitive impairment in the euthymic phase of chronic unipolar depression. J Nerv Ment Dis 1997; $185: 748-54$

22. Mora E, Portella MJ, Forcada I, et al. Persistence of cognitive impairment and its negative impact on psychosocial functioning in lithium-treated, euthymic bipolar patients: a 6-year follow-up study. Psychol Med 2013;43:1187-96.

23. Jaeger J, Vieta E. Functional outcome and disability in bipolar disorders: ongoing research and future directions. Bipolar Disord 2007;9:1-2.

24. Gildengers AG, Butters MA, Chisholm D, et al. Cognitive functioning and instrumental activities of daily living in late-life bipolar disorder. Am J Geriatr Psychiatry 2007:15:174-9.

25. Dickerson FB, Boronow JJ, Stallings $\mathrm{CR}$, et al. Association between cognitive functioning and employment status of persons with bipolar disorder. Psychiatr Serv 2004;55:54-8.

26. Depp CA, Mausbach BT, Eyler LT, et al. Performance-based and subjective measures of functioning in middle-aged and older adults with bipolar disorder. J Nerv Ment Dis 2009;197:471-5.

27. Bowie CR, Depp C, McGrath JA, et al. Prediction of real-world functional disability in chronic mental disorders: a comparison of schizophrenia and bipolar disorder. Am J Psychiatry 2010;167:1116-24.

28. Altshuler LL, Bearden CE, Green MF, et al. A relationship between neurocognitive impairment and functional impairment in bipolar disorder: a pilot study. Psychiatry Res 2008;157:289-93.

29. Altshuler L, Tekell J, Biswas K, et al. Executive function and employment status among veterans with bipolar disorder. Psychiatr Serv 2007;58:1441-7.

30. Alfonso JP, Caracuel A, Delgado-Pastor LC, et al. Combined Goa Management Training and Mindfulness meditation improve executive functions and decision-making performance in abstinent polysubstance abusers. Drug Alcohol Depend 2011;117:78-81.

31. Trivedi MH, Greer TL. Cognitive dysfunction in unipolar depression: implications for treatment. J Affect Disord 2014;152-154:19-27.

32. Gildengers A, Price J, Meryl B, et al. Neuroprotective effects of long-term lithium treatment on amyloid deposition in older adults with bipolar disorder. Neuropsychopharmacology 2012;38:S415.

33. Gray SL, Anderson ML, Dublin S, et al. Cumulative use of strong anticholinergics and incident dementia: a prospective cohort study. JAMA Intern Med 2015;175:401-7.

34. Barnes DE, Yaffe K. The projected effect of risk factor reduction on Alzheimer's disease prevalence. Lancet Neurol 2011;10:819-28.

35. Meusel LA, Hall GB, Fougere $P$, et al. Neural correlates of cognitive remediation in patients with mood disorders. Psychiatry Res 2013;214:142-52.

36. Elgamal S, McKinnon MC, Ramakrishnan K, et al. Successful computer-assisted cognitive remediation therapy in patients with unipolar depression: a proof of principle study. Psychol Med 2007;37:1229-38

37. Taylor V, MacQueen G. Associations between bipolar disorder and metabolic syndrome: a review. J Clin Psychiatry 2006;67:1034-41.

38. Kiecolt-Glaser JK, Glaser R. Depression and immune function central pathways to morbidity and mortality. J Psychosom Res 2002;53:873-6.
39. Bryan J, Tiggemann M. The effect of weight-loss dieting on cognitive performance and psychological well-being in overweight women. Appetite 2001;36:147-56

40. Ionut V, Bergman RN. Mechanisms responsible for excess weight loss after bariatric surgery. J Diabetes Sci Technol 2011;5: 1263-82.

41. Oldfield RC. The assessment and analysis of handedness: the Edinburgh inventory. Neuropsychologia 1971;9:97-113.

42. Hamilton. SJsH. Bariatric Surgery Program. Secondary Bariatric Surgery Program, 2015. http://www.stjoes.ca/default.asp? action=article $\& \mid \mathrm{D}=1612$

43. Buchwald H, Oien DM. Metabolic/bariatric surgery worldwide 2008 . Obes Surg 2009;19:1605-11.

44. Scopinaro NMD. Biliopancreatic diversion: mechanisms of action and long-term results. Obes Surg 2006;16:683-9.

45. Hedlung JL, Vieweg BW. The Hamilton rating scale for depression: a comprehensive review. J Operational Psychiatry 1979;10: 149-65.

46. Young RC, Biggs JT, Ziegler VE, et al. A rating scale for mania: reliability, validity and sensitivity. Br J Psychiatry 1978;133:429-35.

47. Beck AT, Steer RA, Brown GK. Manual for Beck Depression Inventory II (BDI II). San Antonio, Texas: Psychology Corporation, 1996.

48. Altman EG, Hedeker D, Peterson JL, et al. The Altman self-rating mania scale. Biol Psychiatry 1997;42:948-55.

49. Midei AJ, Matthews KA. Interpersonal violence in childhood as a risk factor for obesity: a systematic review of the literature and proposed pathways. Obes Rev 2011;12:e159-72.

50. Athanasos $P$, Neild R, De Crespigny $C$. The impact of childhood trauma: preliminary findings. Aust Nurs $J$ 2010;18:38-40.

51. Bernstein DP, Stein JA, Newcomb MD, et al. Development and validation of a brief screening version of the childhood trauma questionnaire. Child Abuses \& Neglect 2003;27:169-190

52. Fitzpatrick S, Gilbert S, Serpell L. Systematic review: are overweight and obese individuals impaired on behavioural tasks of executive functioning? Neuropsychol Rev 2013;23:138-56.

53. Boeka AG, Lokken KL. Neuropsychological performance of a clinical sample of extremely obese individuals. Arch Clin Neuropsychol 2008:23:467-74.

54. Lokken KL, Boeka AG, Yellumahanthi K, et al. Cognitive performance of morbidly obese patients seeking bariatric surgery. Am Surg 2010;76:55-9.

55. Wolf PA, Beiser A, Elias MF, et al. Relation of obesity to cognitive function: importance of central obesity and synergistic influence of concomitant hypertension. The Framingham heart study. Curr Alzheimer Res 2007;4:111-16.

56. Galioto R, King WC, Bond DS, et al. Physical activity and cognitive function in bariatric surgery candidates. Int $\mathrm{J}$ Neurosci 2014;124:912-18.

57. Cserjesi R, Molnar D, Luminet $\mathrm{O}$, et al. Is there any relationship between obesity and mental flexibility in children? Appetite 2007;49:675-8

58. Strauss E, Sherman EMS, Spreen O. A compendium of neuropsychological tests. 3rd edn. New York, NY: Oxford University Press, 2006

59. Bove RM, Brick DJ, Healy BC, et al. Metabolic and endocrine correlates of cognitive function in healthy young women. Obesity (Silver Spring) 2013;21:1343-9.

60. Dore GA, Elias MF, Robbins MA, et al. Relation between central adiposity and cognitive function in the Maine-Syracuse study: attenuation by physical activity. Ann Behav Med 2008;35:341-50.

61. Volkow ND, Wang GJ, Fowler JS, et al. Overlapping neuronal circuits in addiction and obesity: evidence of systems pathology. Philos Trans R Soc Lond B Biol Sci 2008;363:3191-200.

62. Fagundo $A B$, de la Torre R, Jimenez-Murcia $S$, et al. Executive functions profile in extreme eating/weight conditions: from anorexia nervosa to obesity. PLoS ONE 2012;7:e43382.

63. Nilsson LG, Nilsson E. Overweight and cognition. Scand J Psychol 2009;50:660-7.

64. Delis DC, Kramer JH, Kaplan E, et al. California verbal learning test: adult version. San Antonio, Texas: Harcourt Brace \& Company, 1987.

65. Wechsler D. Wechsler Memory Scale. 3rd edn. San Antonio, TX: The Psychological Association, 1997

66. Benedict RHB. Brief Visuospatial Memory Test-Revised: manual. Odessa, Florida: Psychological Assessment Resources, 1997.

67. Benton AL, Hamsher K, Sivan AB. Multilingual Aphasia Examination. 3rd edn. lowa City, lowa: AJA Associates, 1983.

68. Golden JC. Stroop Color and Word Test. Chicago, Illinois: Stoelting Company, 1978. 
69. Heaton EK, Chelune GJ, Talley JL, et al. Wisconsin Card Sorting Test (WCST) manual revised and expanded. Odessa, Florida: Psychological Assessment Resources, 1993.

70. Reitan RM, Wolfson D. The halstead-reitan neuropsychological test battery. Tucson, AZ: Neuropsychology Press, 1985.

71. Gronwall D. Paced auditory serial-addition task: a measure of recovery form concussion. Percept Mot Skills 1977:44:367-73.

72. Wechsler D. Wechsler Abbreviated Scale of Intelligence (WASI). San Antonio, TX: NCS Pearson Inc., 1999.

73. Wechsler D. Wechsler Test of Adult Reading: WTAR. San Antonio, TX: The Psychological Corporation, 2001.

74. WHO. WHO STEPwise approach to surveillance (STEPS). Geneva, Switzerland: World Health Organization (WHO), 2008.

75. Chung F, Yegneswaran B, Liao P, et al. Validation of the Berlin Questionnaire and American Society of Anesthesiologists checklist as screening tools for obstructive sleep apnea in surgical patients. Anesthesiology 2008;108:822-30.

76. Heyn P, Abreu BC, Ottenbacher KJ. The effects of exercise training on elderly persons with cognitive impairment and dementia: a meta-analysis. Arch Phys Med Rehabil 2004;85:1694-704.

77. Olson AK, Eadie BD, Ernst C, et al. Environmental enrichment and voluntary exercise massively increase neurogenesis in the adult hippocampus via dissociable pathways. Hippocampus 2006;16:250-60.

78. Willett WC, Leibel RL. Dietary fat is not a major determinant of body fat. Am J Med 2002;113:47-59.

79. Basiotis PP, Welsh SO, Cronin FJ, et al. Number of days of food intake records required to estimate individual and group nutrient intakes with defined confidence. J Nutr 1987;117:1638-41.

80. Sheehan DV, Harnett-Sheehan K, Raj BA. The measurement of disability. Int Clin Psychopharmacol 1996;11(Suppl 3):89-95.

81. Broadbent DE, Cooper PF, FitzGerald $P$, et al. The cognitive failures questionnaire (CFQ) and its correlates. Br J Clin Psychol 1982;21 (Pt 1):1-16.

82. Yim CY, Soczynska JK, Kennedy SH, et al. The effect of overweight/ obesity on cognitive function in euthymic individuals with bipolar disorder. Eur Psychiatry 2012;27:223-8.

83. Warrington EK. Recognition Memory Test. Windsor, UK: NFER-Nelson, 1984.

84. Thirion B, Pinel P, Meriaux S, et al. Analysis of a large fMRI cohort: statistical and methodological issues for group analyses. Neuroimage 2007;35:105-20.

85. Woods RP. Modeling for intergroup comparisons of imaging data. Neuroimage 1996;4(3Pt 3):S84-94. 